\title{
Infrared finite effective charge of QCD
}

\author{
A. C. Aguilar, ${ }^{a}$ D. Binosi ${ }^{b}$ and J. Papavassiliou ${ }^{\star a}$ \\ ${ }^{a}$ Departamento de Física Teórica and IFIC, Centro Mixto, Universidad de Valencia-CSIC \\ E-46100, Burjassot, Valencia, Spain \\ ${ }^{b}$ European Centre for Theoretical Studies in Nuclear Physics and Related Areas (ECT*), \\ Villa Tambosi, Strada delle Tabarelle 286, I-38050 Villazzano (TN), Italy. \\ E-mail: Arlene.Aguilar@uv.es,binosi@ect.it, \\ Joannis.Papavassiliou@uv.es
}

\begin{abstract}
We show that the gauge invariant treatment of the Schwinger-Dyson equations of QCD leads to an infrared finite gluon propagator, signaling the dynamical generation of an effective gluon mass, and a non-enhanced ghost propagator, in qualitative agreement with recent lattice data. The truncation scheme employed is based on the synergy between the pinch technique and the background field method. One of its most powerful features is that the transversality of the gluon self-energy is manifestly preserved, exactly as dictated by the BRST symmetry of the theory. We then explain, for the first time in the literature, how to construct non-perturbatively a renormalization group invariant quantity out of the conventional gluon propagator. This newly constructed quantity serves as the natural starting point for defining a non-perturbative effective charge for QCD, which constitutes, in all respects, the generalization in a non-Abelian context of the universal QED effective charge. This strong effective charge displays asymptotic freedom in the ultraviolet, while in the low-energy regime it freezes at a finite value, giving rise to an infrared fixed point for QCD. Some possible pitfalls related to the extraction of such an effective charge from infrared finite gluon propagators, such as those found on the lattice, are briefly discussed.
\end{abstract}

LIGHT CONE 2008 Relativistic Nuclear and Particle Physics

July 7-11 2008

Mulhouse, France

${ }^{*}$ Speaker. 
It has been known for a long time that even though the gluon is massless at the level of the fundamental Lagrangian, and remains massless to all order in perturbation theory, the non-perturbative QCD dynamics generate an effective, momentum-dependent mass, without affecting the local $S U(3)_{c}$ invariance, which remains intact [1]. The existence of this mass is discovered by studying the Schwinger-Dyson equations (SDEs) of QCD, in a gauge-invariant framework known as the pinch technique (PT) $[1,2]$. To obtain massive solutions it is necessary to include longitudinallycoupled massless scalars in the Green's functions, which play a role rather like Goldstone excitations, but do not signal any sort of breakdown of local gauge symmetry, which is preserved. Like standard Goldstone bosons these massless scalars do not appear explicitly in the $S$-matrix; however, they play a crucial role in confinement, furnishing the required long range potential.

An effective low-energy field theory for describing the gluon mass is the gauged non-linear sigma model known as "massive gauge-invariant Yang-Mills" [3], with Lagrangian density

$$
\mathscr{L}_{M Y M}=\frac{1}{2} G_{\mu \nu}^{2}-m^{2} \operatorname{Tr}\left[A_{\mu}-g^{-1} U(\theta) \partial_{\mu} U^{-1}(\theta)\right]^{2},
$$

where $A_{\mu}=\frac{1}{2 i} \sum_{a} \lambda_{a} A_{\mu}^{a}$, the $\lambda_{a}$ are the SU(3) generators (with $\operatorname{Tr} \lambda_{a} \lambda_{b}=2 \delta_{a b}$ ), and the $N \times N$ unitary matrix $U(\theta)=\exp \left[i \frac{1}{2} \lambda_{a} \theta^{a}\right]$ describes the scalar fields $\theta_{a}$. Note that $\mathscr{L}_{M Y M}$ is locally gauge-invariant under the combined gauge transformation

$$
A_{\mu}^{\prime}=V A_{\mu} V^{-1}-g^{-1}\left[\partial_{\mu} V\right] V^{-1}, \quad U^{\prime}=U\left(\theta^{\prime}\right)=V U(\theta)
$$

for any group matrix $V=\exp \left[i \frac{1}{2} \lambda_{a} \omega^{a}(x)\right]$, where $\omega^{a}(x)$ are the group parameters. One might think that, by employing (2), the fields $\theta_{a}$ can always be transformed to zero, but this is not so if the $\theta_{a}$ contain vortices. To use the $\mathscr{L}_{M Y M}$ in (1), one solves the equations of motion for $U$ in terms of the gauge potentials and substitutes the result in the equations for the gauge potential. One then finds the Goldstone-like massless modes mentioned above. This model admits vortex solutions [3], with a long-range pure gauge term in their potentials, which endows them with a topological quantum number corresponding to the center of the gauge group $\left[Z_{N}\right.$ for $\left.S U(N)\right]$, and is, in turn, responsible for quark confinement and gluon screening. Specifically, center vortices of thickness $\sim m^{-1}$, where $m$ is the induced mass of the gluon, form a condensate because their entropy (per unit size) is larger than their action. This condensation furnishes an area law to the fundamental representation Wilson loop, thus confining quarks $[1,3]$.

Of course $\mathscr{L}_{M Y M}$ is not renormalizable, and breaks down in the ultraviolet. This breakdown simply reflects the fact that the gluon mass $m$ in (1) is assumed to be constant, while the solutions of the SDEs reveal that the mass is momentum-dependent, vanishing at large $q^{2}$ [1]. Specifically, when studying the SDE for the gluon propagator, $\Delta\left(q^{2}\right)$, one looks for infrared finite solutions, i.e. with $\Delta^{-1}(0)>0$. Such solutions may be fitted by "massive" propagators of the form $\Delta^{-1}\left(q^{2}\right)=q^{2}+m^{2}\left(q^{2}\right)$, where $m^{2}\left(q^{2}\right)$ is not "hard", but depends non-trivially on the momentum transfer $q^{2}$. When the renormalization-group logarithms are properly taken into account in the SDE analysis, one obtains, in addition, the non-perturbative generalization of $\alpha\left(q^{2}\right)$, the QCD running coupling (effective charge). The presence of $m^{2}\left(q^{2}\right)$ in the argument of $\alpha\left(q^{2}\right)$ tames the Landau singularity associated with the perturbative $\beta$ function, and the resulting effective charge is asymptotically free in the ultraviolet , "freezing" at a finite value in the infrared. 
The general picture described above has received spectacular confirmation from a plethora of lattice studies, spanning a period of several years: the gluon propagator reaches indeed a finite (nonvanishing) value in the deep infrared, as predicted by Cornwall. This rather characteristic behavior was already seen in early studies [4], and has been firmly established recently using large-volume lattices, for both $S U(2)$ [5] and $S U(3)$ [6] pure Yang-Mills (no quarks included).

In this talk we will present recent results from a gauge-invariant study of the coupled gluonghost system of SDEs [7], yielding an infrared finite gluon propagator and a divergent (but nonenhanced) ghost propagator, in qualitative agreement with recent lattice data [5, 6]; this behavior has also been confirmed within the Gribov-Zwanziger formalism [8]. As the title suggests, we will eventually focus on the issue of the infrared finite QCD effective charge.

Obtaining an infrared finite result for the gluon self-energy from SDEs, without violating the underlying local gauge symmetry, is far from trivial, and hinges crucially on one's ability to devise a self-consistent truncation scheme that would select a tractable and, at the same time, physically meaningful subset of these equations. Specifically, while in QED the Green's functions satisfy naive Ward Identities (WIs), in QCD they satisfy complicated Slavnov-Taylor identities (STIs), which involve various composite ghost operators. To see how this complicates the truncation procedure of the SDEs, consider the STI of the gluon self-energy

$$
q^{\mu} \Pi_{\mu v}(q)=0 .
$$

Eq. (3) is without a doubt the most fundamental statement at the level of Green's functions that one can obtain from the BRST symmetry; it affirms the transversality of the gluon self-energy and is valid both perturbatively to all orders as well as non-perturbatively. The problem is that in the SDE governing $\Pi_{\mu v}(q)$ enter higher order Green's functions, namely the fully-dressed fundamental vertices of the theory, which satisfy complicated STIs. Thus, whereas in QED the validity of Eq. (3) can be easily seen at the level of the SDE, simply because $q^{\mu} \Gamma_{\mu}(p, p+q)=e\left[S^{-1}(p+q)-S^{-1}(p)\right]$, in QCD proving Eq. (3) is very difficult, and requires the conspiracy of all full vertices appearing in the SDE. Truncating the SDE naively usually amounts to leaving out some of these vertices, and, as a result, Eq. (3) is compromised. Instead, the gauge-invariant truncation scheme [9], based on the PT $[1,2]$ and its correspondence with the background field method (BFM) [10] maintains the validity of Eq.(3) at every level of approximation.

The gluon propagator in the covariant gauges has the form $i \Delta_{\mu v}(q)=\left[\mathrm{P}_{\mu v}(q) \Delta\left(q^{2}\right)+\xi \frac{q_{\mu} q_{v}}{q^{4}}\right]$, where $\xi$ denotes the gauge-fixing parameter, $\mathrm{P}_{\mu v}(q)=g_{\mu \nu}-q_{\mu} q_{v} / q^{2}$ is the usual transverse projector, and, finally, $\Delta^{-1}\left(q^{2}\right)=q^{2}+i \Pi\left(q^{2}\right)$, with $\Pi_{\mu v}(q)=\mathrm{P}_{\mu v}(q) \Pi\left(q^{2}\right)$ the gluon self-energy. The full ghost propagator $D\left(p^{2}\right)$ and its self-energy $L\left(p^{2}\right)$ are related by $i D^{-1}\left(p^{2}\right)=p^{2}-i L\left(p^{2}\right)$. In the case of pure (quarkless) QCD, the new SD series [9] for the gluon and ghost propagators reads (see also Fig. 1)

$$
\begin{aligned}
& \Delta^{-1}\left(q^{2}\right) \mathrm{P}_{\mu v}(q)=\frac{q^{2} \mathrm{P}_{\mu v}(q)+i \sum_{i=1}^{4}\left(a_{i}\right)_{\mu v}}{\left[1+G\left(q^{2}\right)\right]^{2}}, \\
& i D^{-1}\left(p^{2}\right)=p^{2}+i \lambda \int_{k} \Gamma^{\mu} \Delta_{\mu v}(k) \Gamma^{v}(p, k) D(p+k), \\
& i \Lambda_{\mu v}(q)=\lambda \int_{k} H_{\mu \rho}^{(0)} D(k+q) \Delta^{\rho \sigma}(k) H_{\sigma v}(k, q),
\end{aligned}
$$



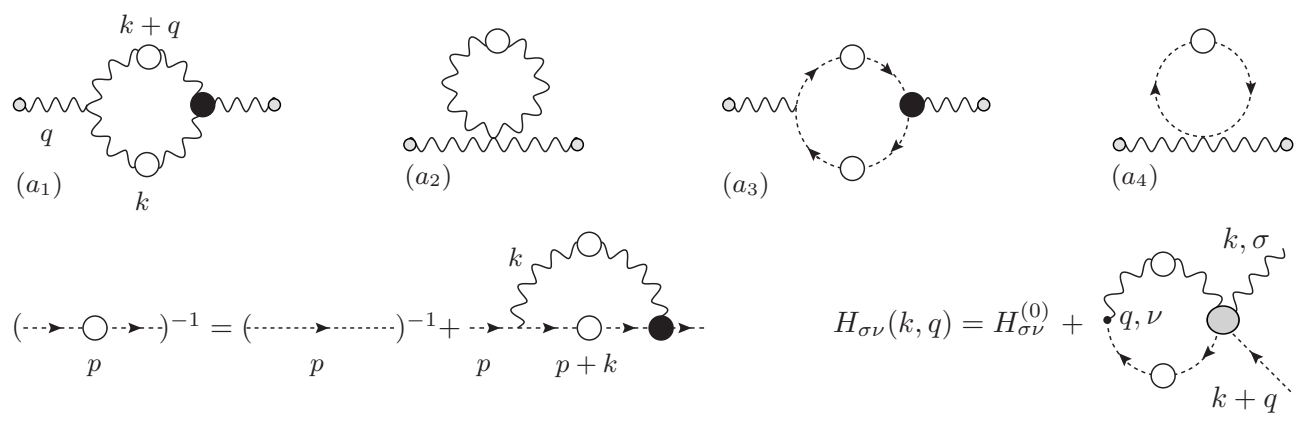

Figure 1: The PT-BFM gluon-ghost system. The circles attached to the external gluons denote that, from the point of view of Feynman rules, they are treated as background fields.

where $\lambda=g^{2} C_{\mathrm{A}}$, with $C_{\mathrm{A}}$ the Casimir eigenvalue of the adjoint representation $\left[C_{\mathrm{A}}=N\right.$ for $\left.S U(N)\right]$, and $\int_{k} \equiv \mu^{2 \varepsilon}(2 \pi)^{-d} \int d^{d} k$, with $d=4-\varepsilon$ the dimension of space-time. $\Gamma_{\mu}$ is the standard (asymmetric) gluon-ghost vertex at tree-level, and $\mathbb{\Gamma}^{v}$ the fully-dressed one. $G\left(q^{2}\right)$ is the $g_{\mu v}$ component of the auxiliary two-point function $\Lambda_{\mu v}(q)$, and the function $H_{\sigma v}$ is defined diagrammatically in Fig. 1. $H_{\sigma v}$ is related to the full gluon-ghost vertex by $q^{\sigma} H_{\sigma v}(p, r, q)=-i \Gamma_{v}(p, r, q)$; at tree-level, $H_{\sigma \nu}^{(0)}=i g_{\sigma \nu}$. When evaluating the diagrams $\left(a_{i}\right)$ we use the BFM Feynman rules [10]; the BFM fully dressed three-gluon and gluon-ghost vertices are denoted by $\widetilde{\mathbb{\Gamma}}_{\mu \alpha \beta}$ and $\widetilde{\boldsymbol{\Gamma}}_{\mu}$.

Notice a point of paramount importance: due to the Abelian all-order WIs that these two full vertices satisfy (for all $\xi$ ), namely

$$
q^{\mu} \widetilde{\boldsymbol{\Gamma}}_{\mu \alpha \beta}=i \Delta_{\alpha \beta}^{-1}(k+q)-i \Delta_{\alpha \beta}^{-1}(k), \quad q^{\mu} \widetilde{\boldsymbol{\Gamma}}_{\mu}=i D^{-1}(k+q)-i D^{-1}(k),
$$

one can demonstrate that $q^{\mu}\left[\left(a_{1}\right)+\left(a_{2}\right)\right]_{\mu \nu}=0$ and $q^{\mu}\left[\left(a_{3}\right)+\left(a_{4}\right)\right]_{\mu \nu}=0$ [11]. Thus, unlike other treatments in the literature, within this formalism the transversality of the gluon self-energy, i.e. Eq. (3), is preserved at every step, in absolute compliance with the BRST symmetry.

Next, following standard techniques, we express $\widetilde{\boldsymbol{\Gamma}}_{\mu \alpha \beta}$ and $\widetilde{\boldsymbol{\Gamma}}_{\mu}$ as a function of the gluon and ghost self-energy, respectively, in such a way as to automatically satisfy the crucial WIs of Eq. (5); failure to satisfy these WIs would invariably compromise the transversality of the answer. The Ansatz we will use is

$$
\widetilde{\boldsymbol{\Gamma}}_{\mu \alpha \beta}=\Gamma_{\mu \alpha \beta}+i \frac{q_{\mu}}{q^{2}}\left[\Pi_{\alpha \beta}(k+q)-\Pi_{\alpha \beta}(k)\right], \quad \widetilde{\boldsymbol{\Gamma}}_{\mu}=\widetilde{\Gamma}_{\mu}-i \frac{q_{\mu}}{q^{2}}[L(k+q)-L(k)] ;
$$

its essential feature, other than satisfying the aforementioned WIs, is the presence of massless, longitudinally coupled pole terms, which are instrumental for obtaining $\Delta^{-1}(0) \neq 0$ [12]. These poles are not kinematic but dynamical, corresponding to a composite (bound-state) Goldstone excitation, enforcing the local gauge invariance. For the conventional ghost-gluon vertex $\mathbb{\Gamma}_{v}$, appearing in the second SDE of (4) we will use its tree-level expression, i.e., $\boldsymbol{\Gamma}_{v} \rightarrow \Gamma_{v}=-p_{v}$; this is perfectly legitimate, since in this formalism the two ghost vertices, $\mathbb{\Gamma}_{v}$ and $\widetilde{\mathbb{\Gamma}}_{\mu}$, are different. Finally, for $H_{\sigma v}$ we use its tree-level value, $H_{\sigma v}^{(0)}$.

In Fig.2, we show the numerical result for $\Delta\left(q^{2}\right)$ renormalized at $\mu=M_{b}=4.5 \mathrm{GeV}$, and the comparison with the corresponding lattice data of Ref.[6]. In the right panel of Fig.2, we present the dressing function for the ghost propagator, renormalized at the same point. While the infrared 
behavior of the ghost dressing function is in qualitative agreement with the lattice data (no powerlaw enhancement [13]), there is a significant quantitative discrepancy (more than a factor of two), mainly due to the standard approximation $\mathbb{\Gamma}_{v} \rightarrow-p_{v}$, motivated by the ultraviolet finiteness of $\mathbb{\Gamma}_{v}$ in the Landau gauge. A more sophisticated treatment of this vertex, even at the level of perturbation theory, should reduce this difference significantly.
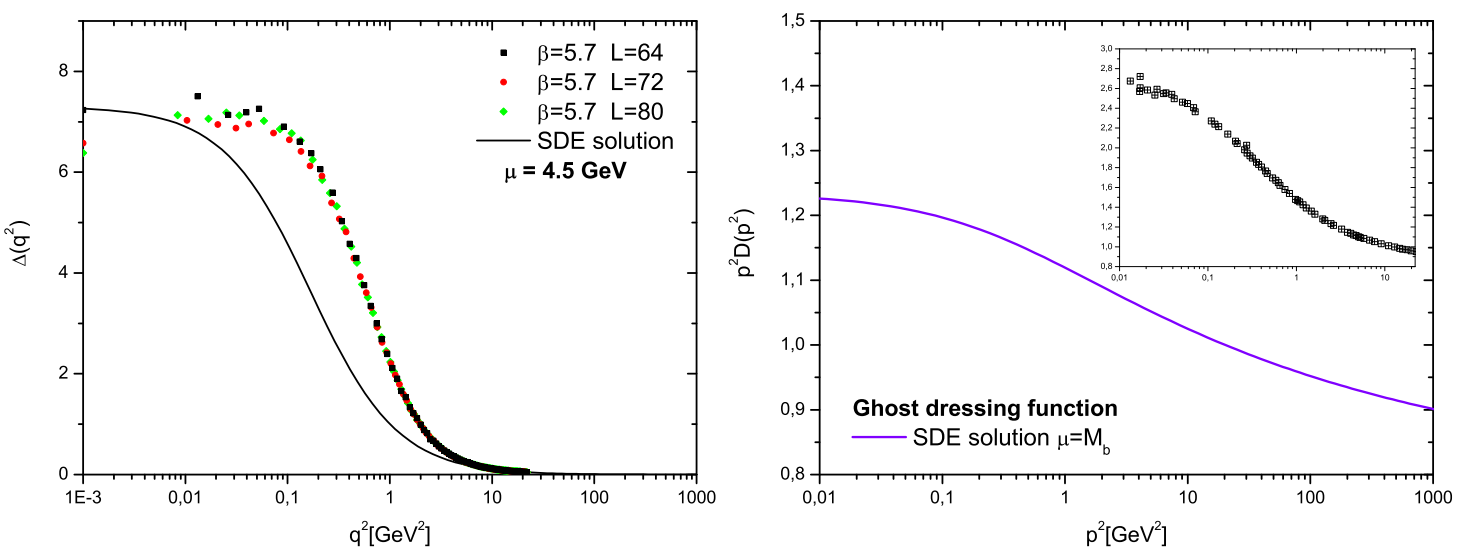

Figure 2: Left Panel:The numerical solution for the gluon propagator from the SDE (black continuous line) compared to the lattice data of Ref.[6]. Right panel:The ghost dressing function $p^{2} D\left(p^{2}\right)$ obtained from the SDE. In the insert we show the lattice data for the same quantity.

Let us now turn to the QCD effective charge. There are two main issues: (i) how to define it consistently at the level of perturbation theory: specifically, which graphs determine the running, and (ii) how to extend the (whatever) definition one reaches in (i) into the non-perturbative regime.

Point (i) has been addressed exhaustively in the literature [14]: the upshot is that in the context of the PT one may replicate to all-orders in perturbation theory the prototype QED construction of an effective charge. To fix the ideas, the PT one-loop gluon self-energy reads

$$
\widehat{\Delta}^{-1}\left(q^{2}\right)=q^{2}\left[1+b g^{2} \ln \left(\frac{q^{2}}{\mu^{2}}\right)\right]
$$

where $b=11 C_{A} / 48 \pi^{2}$ is the first coefficient of the QCD $\beta$-function. Due to the Abelian WIs satisfied by the PT effective Green's functions, the new propagator-like quantity $\widehat{\Delta}^{-1}\left(q^{2}\right)$ absorbs all the RG-logs, exactly as happens in QED with the photon self-energy. Equivalently, since $Z_{g}$ and $\widehat{Z}_{A}$, the renormalization constants of the gauge-coupling and the effective self-energy, respectively, satisfy the QED relation $Z_{g}=\widehat{Z}_{A}^{-1 / 2}$, the product $\widehat{d}\left(q^{2}\right)=g^{2} \widehat{\Delta}\left(q^{2}\right)$ forms a RG-invariant ( $\mu$-independent) quantity [1]; for large momenta $q^{2}$,

$$
\widehat{d}\left(q^{2}\right)=\frac{\bar{g}^{2}\left(q^{2}\right)}{q^{2}},
$$

where $\bar{g}^{2}\left(q^{2}\right)$ is the RG-invariant effective charge of QCD,

$$
\bar{g}^{2}\left(q^{2}\right)=\frac{g^{2}}{1+b g^{2} \ln \left(q^{2} / \mu^{2}\right)}=\frac{1}{b \ln \left(q^{2} / \Lambda^{2}\right)} .
$$

Let us now come to point (ii): assuming that one has non-perturbative information about the infrared behavior of the conventional gluon propagator $\Delta\left(q^{2}\right)$, how should one extract an effective 


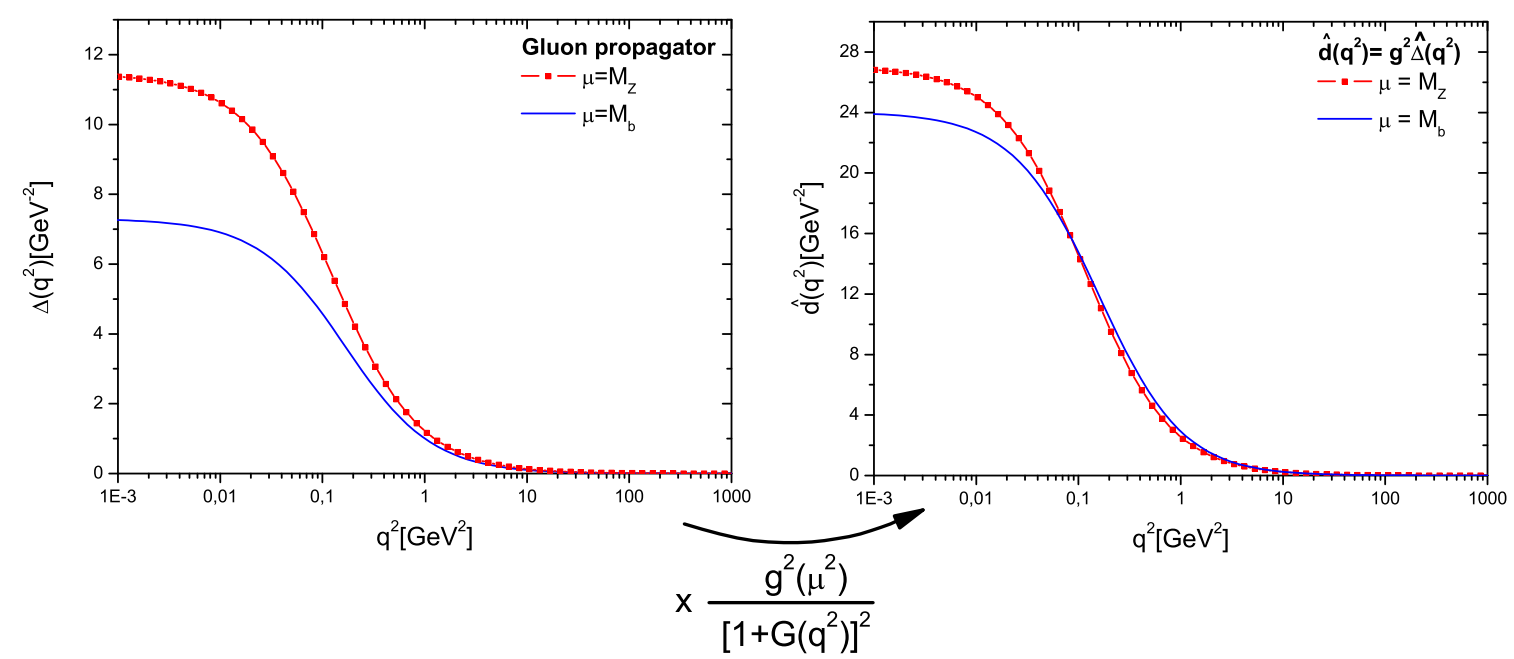

Figure 3: Left panel: The solution of SDE renormalized at $\mu=M_{b}=4.5 \mathrm{GeV}$ (continuous blue curve) and $\mu=M_{Z}=91 \mathrm{GeV}$ (red line+square curve). Right Panel: The corresponding PT-BFM $\widehat{\Delta}\left(q^{2}\right)$ obtained as the convolution of $\Delta\left(q^{2}\right)$ and the function $g^{2}\left(\mu^{2}\right) /\left[1+G\left(q^{2}\right)\right]^{2}$.

charge, which, perturbatively, will go over to Eq. (8)? To accomplish this, one must use an additional field-theoretic ingredient: the conventional $\Delta\left(q^{2}\right)$ and the PT-BFM $\widehat{\Delta}\left(q^{2}\right)$ are related by the formal all-order relation [15]

$$
\Delta\left(q^{2}\right)=\left[1+G\left(q^{2}\right)\right]^{2} \widehat{\Delta}\left(q^{2}\right) .
$$

Note that the $G\left(q^{2}\right)$ already appears in Eq. (4) and Fig.1. With our approximations its SDE reads

$$
G\left(q^{2}\right)=-\frac{\lambda}{3} \int_{k}\left[2+\frac{(k \cdot q)^{2}}{k^{2} q^{2}}\right] \Delta(k) D(k+q) .
$$

First of all, it is easy to verify that at lowest order the $G\left(q^{2}\right)$ obtained from Eq. (10) restores the $\beta$ function coefficient in front of ultraviolet logarithm. In that limit $1+G\left(q^{2}\right)=1+\frac{9}{4} \frac{C_{\mathrm{A}} g^{2}}{48 \pi^{2}} \ln \left(q^{2} / \mu^{2}\right)$ and $\Delta^{-1}\left(q^{2}\right)=q^{2}\left[1+\frac{13}{2} \frac{C_{\mathrm{A}} g^{2}}{48 \pi^{2}} \ln \left(q^{2} / \mu^{2}\right)\right]$. Then using Eq. (9) we recover the $\widehat{\Delta}^{-1}\left(q^{2}\right)$ of Eq. (6), as we should. Then, non-perturbatively, one substitutes into Eq. (9) the $G\left(q^{2}\right)$ and $\Delta\left(q^{2}\right)$ obtained from solving the system in Eq. (4), to obtain $\widehat{\Delta}\left(q^{2}\right)$. This latter quantity is the non-perturbative generalization of Eq. (6); for the same reasons explained above, when multiplied by $g^{2}$ it should form an RG-invariant quantity, e.g. the non-perturbative generalization of $\widehat{d}\left(q^{2}\right)$. In Fig. 3 we present the combined result of the above steps: $\widehat{d}\left(q^{2}\right)$ is obtained from two different sets of solutions of the system Eq. (4), one renormalized at $\mu=M_{b}=4.5 \mathrm{GeV}$ and one at $\mu=M_{Z}=91 \mathrm{GeV}$. Ideally the two curves of $\widehat{d}\left(q^{2}\right)$ should be identical; even though this does not happen, due to the approximations employed when solving the system of Eq. (4), the two curves are fairly close, indicating that $\widehat{d}\left(q^{2}\right)$ is to a very good approximation an RG-invariant quantity, as it should.

We are now in the position to define the non-perturbative QCD effective charge from the RGinvariant quantity $\widehat{d}\left(q^{2}\right)$. Of course, given that $\widehat{d}\left(q^{2}\right)$ reaches a finite value in the deep infrared, it would be completely absurd to define the effective charge by forcing out a factor of $1 / q^{2}$; such a procedure would furnish a completely unphysical strong QCD coupling, namely one that would vanish in the deep infrared(!) where QCD is supposed to be strongly coupled. 


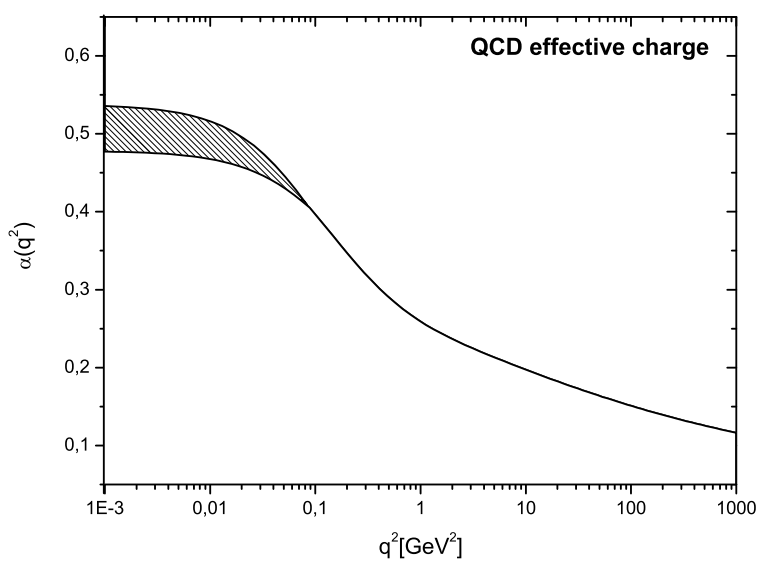

Figure 4: The QCD effective charge, $\alpha\left(q^{2}\right)=\bar{g}^{2}\left(q^{2}\right) / 4 \pi$, extracted from Fig.3 by factoring out a gluon mass of $\mathrm{m}(0)=500 \mathrm{MeV}$.
The correct thing to do is to factor out a "massive" propagator, i.e. write

$$
\widehat{d}\left(q^{2}\right)=\frac{\bar{g}^{2}\left(q^{2}\right)}{q^{2}+m^{2}\left(q^{2}\right)} .
$$

Of course, as we have emphasized, $m^{2}\left(q^{2}\right)$ itself is running, which must also be taken into account in a more sophisticated treatment. For the purposes of this talk, however, we assume that $m^{2}\left(q^{2}\right)$ is constant, $m^{2}\left(q^{2}\right)=m^{2}(0)$, and use for $m(0)$ the value of $500 \mathrm{MeV}$ favored by phenomenology [16]. The $\alpha\left(q^{2}\right)$ obtained is shown in Fig.(4); as announced, at low energies freezes to a finite value, indicating the appearance of an infrared fixed point of QCD.

\section{Acknowledgments}

We thank Prof. J. M. Cornwall for numerous illuminating discussions. Work supported by the Spanish MEC grants FPA 2005-01678 and the Fundación General of the UV.

\section{References}

[1] J. M. Cornwall, Phys. Rev. D 26, 1453 (1982).

[2] J. M. Cornwall and J. Papavassiliou, Phys. Rev. D 40, 3474 (1989).

[3] J. M. Cornwall, Nucl. Phys. B 157, 392 (1979); Phys. Rev. D 57, 7589 (1998).

[4] C. Alexandrou, P. de Forcrand and E. Follana, Phys. Rev. D 63, 094504 (2001),

[5] A. Cucchieri and T. Mendes, PoS LAT2007, 297 (2007).

[6] I. L. Bogolubsky, E. M. Ilgenfritz, M. Muller-Preussker and A. Sternbeck, PoS LAT2007, 290 (2007).

[7] A. C. Aguilar, D. Binosi and J. Papavassiliou, Phys. Rev. D 78, 025010 (2008).

[8] D. Dudal, J. A. Gracey, S. P. Sorella, N. Vandersickel and H. Verschelde, arXiv:0806.4348 [hep-th].

[9] D. Binosi and J. Papavassiliou, Phys. Rev. D 77, 061702 (2008); arXiv:0805.3994 [hep-ph].

[10] L. F. Abbott, Nucl. Phys. B 185, 189 (1981).

[11] A. C. Aguilar and J. Papavassiliou, JHEP 0612, 012 (2006).

[12] R. Jackiw and K. Johnson, Phys. Rev. D 8, 2386 (1973); J. M. Cornwall and R. E. Norton, Phys. Rev. D 8 (1973) 3338; E. Eichten and F. Feinberg, Phys. Rev. D 10, 3254 (1974).

[13] Ph. Boucaud et al., JHEP 0806, 099 (2008); Ph. Boucaud et al., JHEP 0606, 001 (2006).

[14] N. J. Watson, Nucl. Phys. B 494, 388 (1997); D. Binosi and J. Papavassiliou, Nucl. Phys. Proc. Suppl. 121, 281 (2003).

[15] D. Binosi and J. Papavassiliou, Phys. Rev. D 66, 025024 (2002).

[16] A. C. Aguilar, A. Mihara and A. A. Natale, Phys. Rev. D 65, 054011 (2002). 